\title{
Cytochemical differentiation of the axon membrane in A- and C-fibres
}

\author{
S. G. WAXMAN AND D. C. QUICK \\ From the Department of Neurology, Harvard Medical School, Beth Israel Hospital, Boston, and Program \\ in Health Sciences and Technology, and Research Laboratory of Electronics, Massachusetts Institute of \\ Technology, Cambridge, Massachusetts, USA
}

SUMMARY Guinea pig and rat sciatic nerves were fixed with cacodylate-buffered aldehydes and $\mathrm{OsO}_{4}$, and were stained with ferric ion and ferrocyanide. Cytoplasmic surfaces of the non-myelinated nodal axon membrane of A-fibres display distinct electron-dense aggregates of stain. These aggregates were not observed in association with the paranodal or internodal axolemma. The membranes of $\mathrm{C}$-fibres exhibit no staining under these conditions. Thus, the nodal axolemma of normal myelinated fibres is structurally distinct from both the myelinated internodal membrane, and from the axolemma of $\mathrm{C}$-fibres. The ferric ion-ferrocyanide technique may provide a method for marking axonal membrane with normal nodal properties.

In previous studies (Quick and Waxman, 1977) we demonstrated that, under appropriate conditions, ferric ion is bound to the cytoplasmic surface of the axon membrane at nodes of Ranvier in mammalian peripheral myelinated axons. The binding of ferric ion occurred specifically at the nodal axon membrane, and did not occur in internodal regions. These studies indicated that the differences in ion-binding properties are not due to different accessibility of the nodal and internodal axolemma to ferric ion, but rather to differences in structure between the axon membrane at the nodes and the axon membrane in the internodes (Quick and Waxman. 1977). The development of a cytochemical marker for normal nodal membrane would be very useful for developmental and pathological studies on axons, since the degree to which the membrane of the myelinated axon exhibits normal 'nodal' properties during development before the formation of myelin, or in various pathological states in which there are abnormalities of myelin, remains to be determined (see, for example, Rasminsky and Sears, 1972; McDonald, 1974). We have,

This work was supported in part by grants NS-12307, RR-05479, and TO1-EY00090 from the National Institutes of Health, and by a grant from the Bell Telephone Laboratories Inc. Dr Waxman is the recipient of Research Career Development Award KO4-NS-00010 from the National Institute of Neurological and Communicative Disorders and Stroke.

Address for reprint requests: Dr S. G. Waxman, Harvard Neurological Unit. Beth Israel Hospital, 330 Brookline Ave., Boston, Mass. 02215, USA.

Accepted 11 November 1976 therefore, extended our studies on the distribution of ferric ion binding to peripheral nerve axon membranes. In the present paper we show that cytoplasmic ferric ion binding occurs only for A-fibre nodal membrane, and does not occur for C-fibres in the peripheral nervous system.

\section{Materials and methods}

Adult guinea pigs (Hartley strain) and adult rats (Sprague-Dawley strain) were anaesthetised with pentobarbitone, and the sciatic nerves were exposed in the upper leg. The nerves were bathed in situ with a cacodylate-buffered ( $\mathrm{pH} 7.3$ ) solution of $4 \%$ paraformaldehyde and $5 \%$ glutaraldehyde (Karnovsky, $1965,1967)$. After a few minutes bathing in situ, the nerves were excised, immersed in the same fixative, desheathed, and slightly teased. The tissue was washed in cacodylate buffer and post-fixed in cacodylate-buffered $1 \% \mathrm{OsO}_{4}$.

After aldehyde and osmium fixation, the nerve pieces were washed through three changes of distilled water (five minutes each), immersed in $0.01 \mathrm{M} \mathrm{FeCl}_{3}$ for one hour at room temperature, rinsed with distilled water for five minutes, and incubated in $1 \%$ $\mathrm{K}_{4} \mathrm{Fe}(\mathrm{CN})_{6} \cdot 3 \mathrm{H}_{2} \mathrm{O}$ for 20 minutes at room temperature. This staining procedure was adapted from Landon and Langley (1971).

The stained nerves were rinsed once in distilled water, dehydrated in graded alcohol solutions, and embedded in Epon-Araldite. Silver ultrathin sections 
were examined and photographed without further staining in a Jeol $100 \mathrm{~B}$ transmission electron microscope at $60 \mathrm{kV}$.

\section{Results}

Figure 1a shows an electron micrograph of a node of
Ranvier from guinea pig sciatic nerve cut in longitudinal section. The densest precipitates of stain are just below the axon membrane at the node. Some of the stain is also associated with filaments and microtubules in the nodal axoplasm. Internodal and paranodal regions of the axolemma are not stained. Figure $1 b$ shows a node of Ranvier from rat sciatic
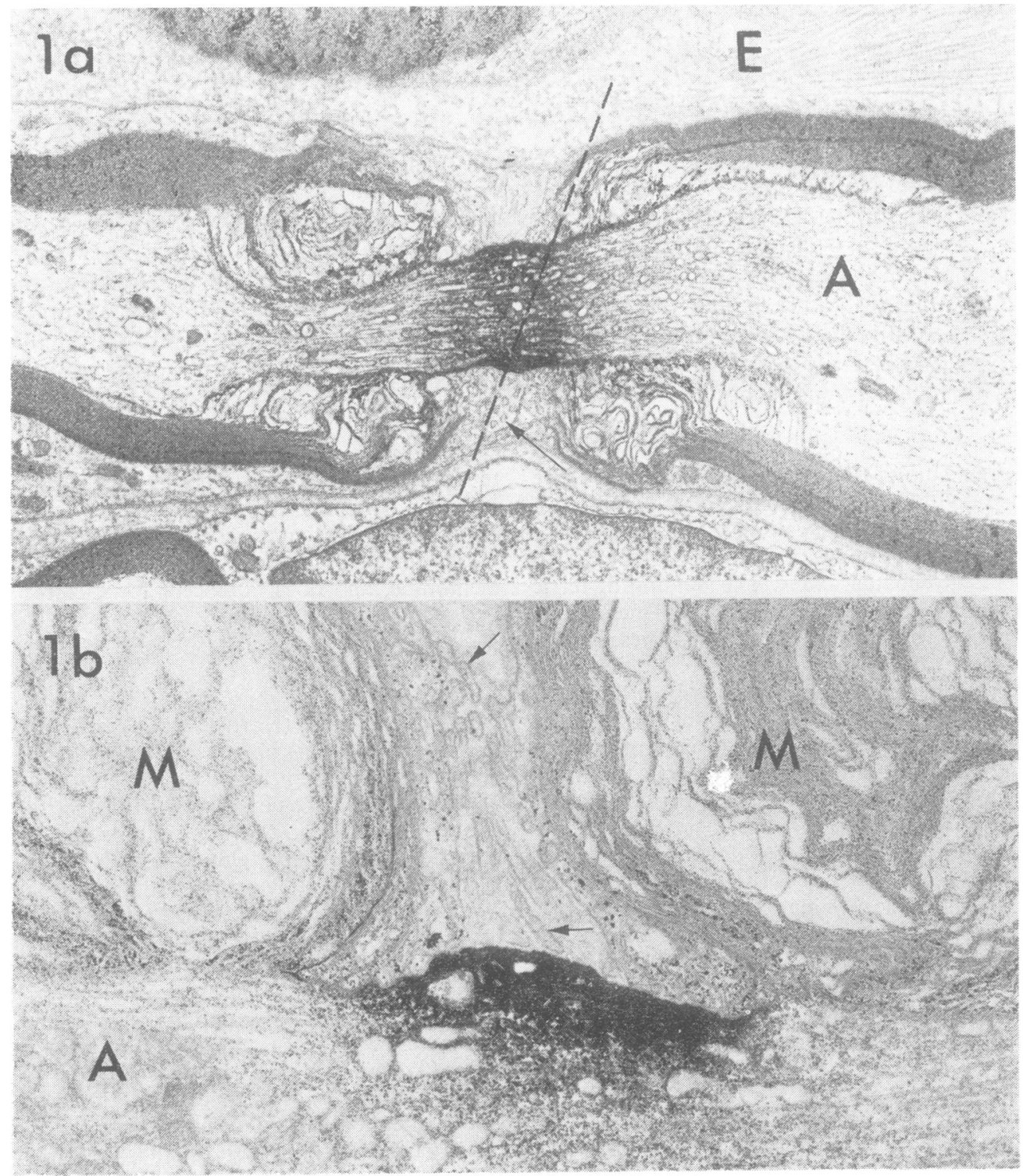

Fig. 1 (a) Longitudinal section of a node of Ranvier (guinea pig) in an A-fibre stained with ferric ion and ferrocyanide. The densest accumulation of stain is in the axoplasm adjacent to the nodal axolemma. The dashed line indicates a plane of section that would give an image similar to that seen in Fig. 2. A: axoplasm; E: extracellular space; arrow: Schwann cell fingers in the nodal gap. $\times 13,000 .($ b $)$ A similar node (rat) at higher magnification. The stain deposit is clearly intra-axonal. A: axoplasm; M: myelin in the region of the terminal loops; arrows: Schwann cell fingers. $\times 40,000$. 
nerve, at a higher magnification. The densest deposits of stain can be seen to be located subjacent to the nodal axolemma. Lesser deposits of electrondense material are associated with the axoplasmic filaments and with the terminating myelin loops, but no stain is present subjacent to the axolemma in the paranodal region. There is no appreciable staining of extracellular components.

Further evidence that this method stains the nodal axon, and not the extracellular component of the node, is shown in Fig. 2, which illustrates, in transverse section, the dense staining of the nodal axon membrane and axoplasmic filaments and tubules, in contrast to the extracellular space between the paranodal Schwann cell fingers, which remains lucent.

C-fibres from guinea pig sciatic nerve, stained under the same conditions, are shown in Fig. 3. Ultrastructural preservation of the C-fibres, using this fixation and staining technique, is good. Their surface membranes are intact, axoplasmic microtubules and filaments are present, and the mitochondria are not swollen. Similarly, the Schwann cells associated with the unmyelinated fibres appear well preserved. In none of the C-fibres is electron-dense precipitate observed subjacent to the axolemma. This is so even for regions of the axon surfaces which are not surrounded by Schwann cell cytoplasm but which are directly accessible to the extracellular milieu (arrows).

Further evidence that the absence of staining of C-fibres is not due to inaccessibility to stain, is shown in Fig. 4. This electron micrograph shows transverse sections of adjacent myelinated and non-myelinated axons. A myelinated fibre sectioned in the internodal region (IA) shows no staining of the axon membrane. One of the myelinated axons (NA) is sectioned at a node of Ranvier. A dense precipitate of stain is present subjacent to the surface membrane of the nodal axon, and is also associated with the axoplasmic filaments at the node. Note that the dense precipitate is not present in the peripheral extracellular

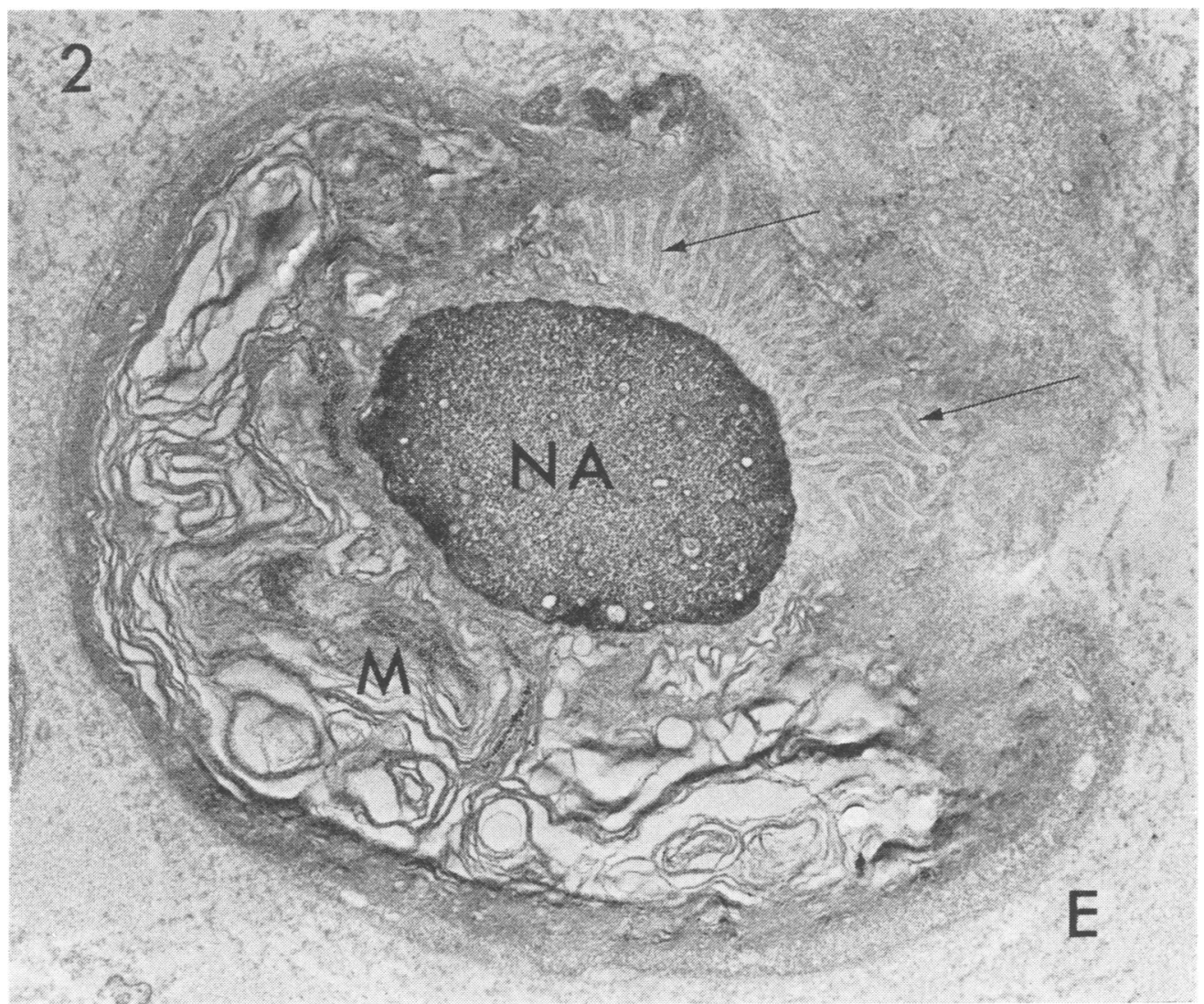

Fig. 2 A transverse section (guinea pig) of a stained node of Ranvier (refer to Fig. la). The densest stain is adjacent to the axolemma. Schwann cell fingers (arrows) and the nodal gap substance that lies between them are not stained. NA: nodal axoplasm; M: paranodal myelin; E: extracellular space. $\times 18,000$. 


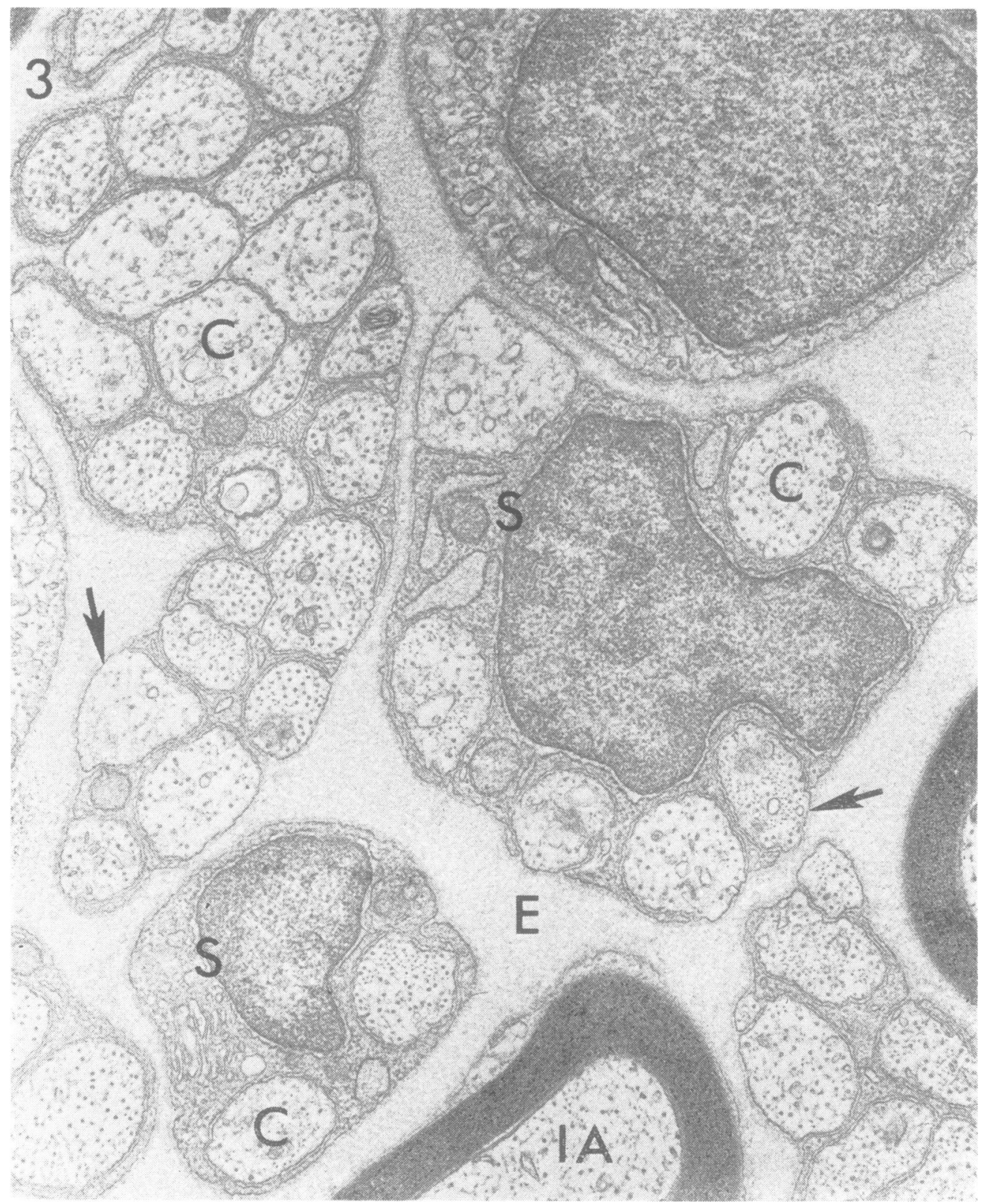

Fig. 3 Transverse section (guinea pig) of C-fibres in tissue that was stained by the ferric ion-ferrocyanide method. This section was cut from the same block as was used for Figs. 1 and 2. The C-fibres are not stained, even where they are exposed to the extracellular space (arrows). C: C-fibres; E: extracellular space; IA: internodal axoplasm of an A-fibre. $\times 26,000$.

space, and that the perinodal Schwann cell processes (arrow) are not seen in negative contrast. In distinct contrast to the axon membrane, the membranes of nearby C-fibres exhibit no precipitates of stain. While, under these fixation and staining conditions, we often observe staining of the inner surface of the nodal $N$ membrane of A-fibres, in no case have we observed staining of C-fibre membranes, in either transverse 


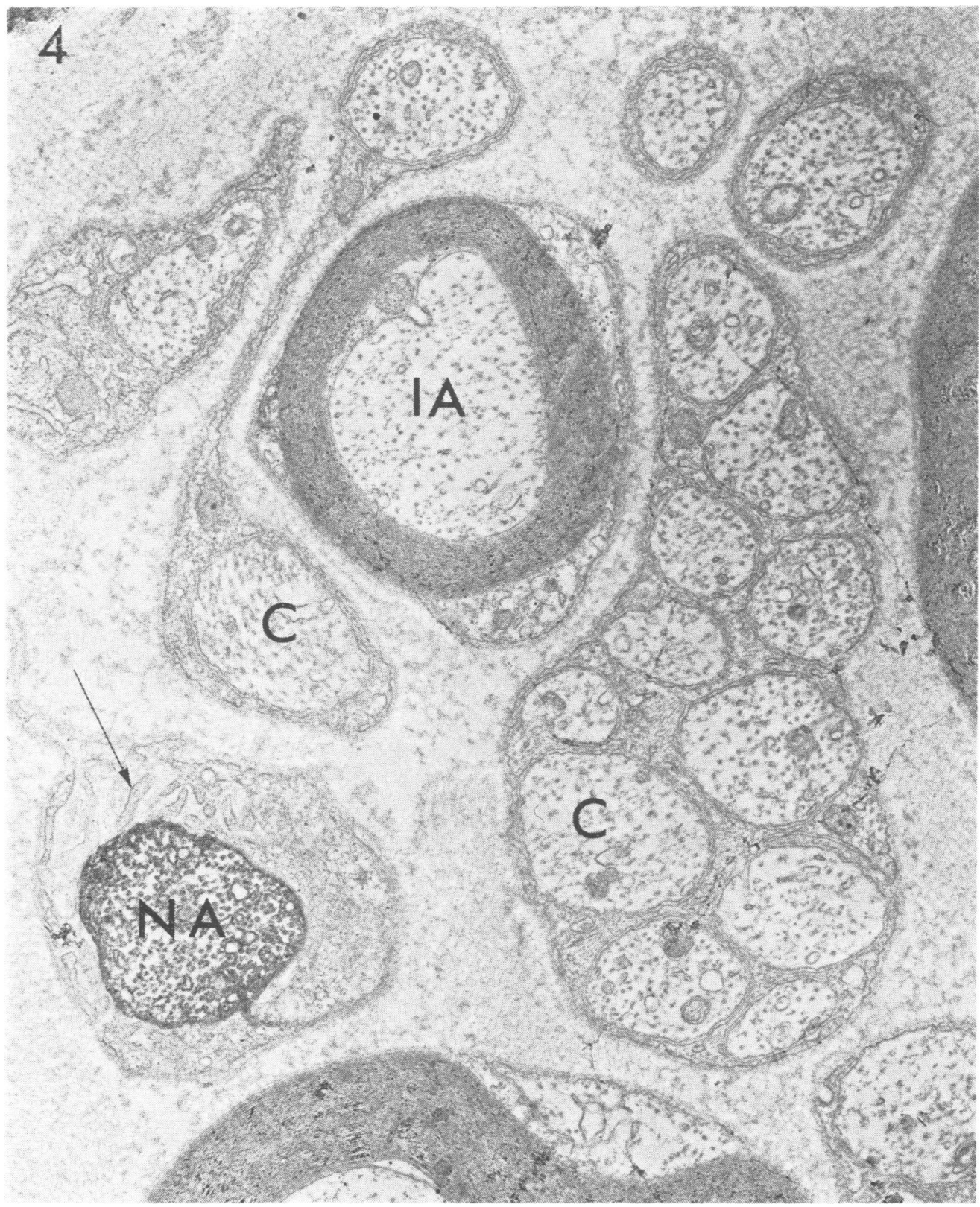

Fig. 4 Transverse section (guinea pig) of C-fibres (C), an internodal axon (IA) of an A-fibre, and a nodal axon (NA) of another A-fibre. The stain is specifically localised in the nodal axoplasm of the A-fibre. Arrow: Schwann cell fingers. $\times 26,000$. 
or longitudinal sections of guinea pig or rat sciatic nerve.

\section{Discussion}

The present results indicate that ferric ion and ferrocyanide stain the inner surface of the non-myelinated nodal axon membrane of mammalian peripheral A.fibres, but not the membranes of C-fibres. In our earlier studies (Quick and Waxman, 1977), we showed that ferric ion specifically stains the cytoplasmic surface of the nodal membrane, but not the internodal or paranodal axon membrane. We also studied (Quick and Waxman, 1977) the specialised electrocyte axons in the gymnotid Sternarchus albifrons, in which there are both excitable and inexcitable nodes of Ranvier (Bennett, 1971; Waxman et al., 1972): and found that only the excitable nodes were stained. This differential staining of nodal and internodal domains of the axon membrane, and of excitable and inexcitable nodes, is consistent with two recent freeze-fracture studies. Rosenbluth (1976) studied amphibian central nodes of Ranvier, and observed a higher density of outer leaflet membrane particles at the nodes than in the internodal axolemma. Kristol et al. (1976) studied the Sternarchus electrocyte axons by freeze-fracture and found a higher density of E-face particles at the excitable nodes than at the inexcitable nodes, which exhibited particle density profiles similar to those of the internodes.

Rasminsky and Sears (1972) showed, in large (internode distance $>0.7 \mu \mathrm{m}$ ) ventral root fibres demyelinated with diphtheria toxin, that conduction remains saltatory to the point of conduction block. Their evidence suggested increased internodal capacitance and transverse conductance in the demyelinated region, but they did not directly examine the electrical properties of the internodal axon membrane. In ventral root axons of dystrophic mice, which are either bare or thinly myelinated, Rasminsky and Kearney (1976) found that adjacent portions of the same axon are capable of sustaining both saltatory and continuous conduction. Ritchie and Rogart (1977) recently estimated the density of sodium channels in mammalian myelinated fibres from measurements of the binding of ${ }^{3} \mathrm{H}$-saxitoxin to rabbit sciatic nerve. Their results suggest a density of approximately $10^{4} / \mu \mathrm{m}^{2}$ for the nodes of Ranvier, and a density of less than $25 / \mu \mathrm{m}^{2}$ for the internodes. Sodium channel density for mammalian C-fibres is approximately $110 / \mu \mathrm{m}^{2}$ (Ritchie et al., 1976).

Our results, as reported here and in our previous paper (Quick and Waxman, 1977), combined with the freeze-fracture data and physiological studies discussed in the preceding paragraphs, point to a rather broad generalisation that the nodal axolemma is

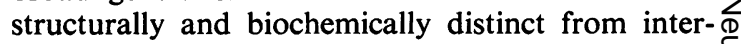
nodal axolemma, from the axolemma of unmyelinated fibres, and from the axolemma of certain $\stackrel{\text { ( }}{\subseteq}$ specialised inexcitable nodes (Waxman et al., 1972). Since our application of the ferric ion-ferrocyanide $D$ technique appears to allow specific staining of normal nodal membrane, we are encouraged to believe that $\frac{}{\mathrm{D}}$. this method may prove to be useful as a method of identifying normal nodal membrane by light and electron microscopy. The degree to which the non- $\overrightarrow{\vec{D}}$ myelinated axon membrane of the developing A-fibre prior to myelination, or the denuded axon membrane $\frac{\bar{O}}{0}$ following demyelination, exhibit the properties of $\overline{\bar{c}}$ normal nodal membrane, remains to be explored. In $\bar{\varnothing}$ this regard, the ferric ion-ferrocyanide staining tech- $\frac{a}{0}$ nique may prove useful in terms of marking the ${ }^{\infty}$ extent of membrane with normal 'nodal' properties $\vec{\circ}$ in the developing nervous system, as well as in the demyelinating and dysmyelinating diseases.

It is a pleasure to thank Miss E. Hartwieg for excellent technical assistance.

\section{References}

Bennett, M. V. L. (1971). Electric organs. In Fi Physiology, Vol. 5, pp. 347-491. Edited by W. S. Hoar and D. J. Randall. Academic Press: New York.

Karnovsky, M. J. (1965). A formaldehyde-glutaraldehy fixative of high osmolality for use in electron micrescopy. Journal of Cell Biology, 27, 137a-138a.

Karnovsky, M. J. (1967). The ultrastructural basis capillary permeability studied with peroxidase as a tracer. Journal of Cell Biology, 35, 213-236.

Kristol, C., Akert, K., Sandri, C., Wyss, U., Bennett, M. V. L., and Moor, H. (1976). The Ranvier nodes in the neurogenic electric organ of the knife fish Stern-0 archus: A freeze-etching study on the distribution of $\mathbb{Q}$ membrane-associated particles. Brain Research (in $\overrightarrow{\vec{O}}$ press).

Landon, D. N. and Langley, O. K. (1971). The local $\frac{\Im}{\sqrt{2}}$ chemical environment of nodes of Ranvier: A study of cation binding. Journal of Anatomy (London), 108, 419-432.

McDonald, W. I. (1974). Remyelination in relation to clinical lesions of the central nervous system. British Medical Bulletin, 30, 186-189.

Quick, D. C. and Waxman, S. G. (1977). Specific staining of the axon membrane at nodes of Ranvier with ferric ion and ferrocyanide. Journal of the Neurological Sciences, 33, 1-11.

Rasminsky, M. and Kearney, R. E. (1976). Continuous $D$ conduction in large diameter bare axons in spinal roots of dystrophic mice. Neurology (Minneap.), 26, 367.

Rasminsky, M. and Sears, T. A. (1972). Internodal 0 S conduction in undissected demyelinated nerve fibres. N Journal of Physiology (London), 227, 323-350.

Ritchie, J. M. and Rogart, R. B. (1977). The density of 
sodium channels in mammalian myelinated nerve fibers and the nature of the axonal membrane under the myelin sheath. Proceedings of the National Academy of Science of the United States, 74, 211-215.

Ritchie, J. M., Rogart, R. B., and Strichartz, G. (1976). A new method for labeling saxitoxin and its binding to non-myelinated fibres of the rabbit vagus, lobster walking, and garfish olfactory nerves. Journal of Physiology (London), 261, 477-494.
Rosenbluth, J. (1976). Structure of electrically excitable membrane at nodes of Ranvier in the frog brain. Journal of Cell Biology, 70, 182a.

Waxman, S. G., Pappas, G. D., and Bennett, M. V. L. (1972). Morphological correlates of functional differentiation of nodes of Ranvier along single fibers in the neurogenic electric organ of the knife fish Sternarchus. Journal of Cell Biology, 53, 210-224. 